\title{
HARMONIC BALANCE APPROACH TO PERIODIC SOLUTIONS OF NONLINEAR JERK EQUATIONS
}

\author{
H. P. W. GOTTLIEB
}

School of Science, Griffith University, Nathan, Queensland 4111, Australia

(Revised, February 2003)

Running Headline: HARMONIC BALANCE AND JERK EQUATIONS

Total Number of Pages: 30 including: 4 Tables over 5 pages. 


\section{Summary}

The method of harmonic balance is applied to nonlinear jerk equations, which involve the third-order time-derivative. For many types of cubic nonlinearities, the method yields good estimates of the period and displacement amplitude of oscillations for a range of values of initial velocity amplitude when compared with numerical solutions. Some limitations, notably the restriction to zero initial acceleration, as well as implications and possible extensions are discussed. 


\section{INTRODUCTION}

The method of harmonic balance (HB), as described for example in the book by Mickens [1] for second-order nonlinear oscillators, can be very successful in predicting the oscillation frequency in terms of the amplitude of periodic solutions to nonlinear "acceleration" equations of the form $\ddot{x}=f(x, \dot{x})$. In its simplest form, the dependent variable is assumed to be a harmonic, cosinusoidal, function of angular frequency $\times$ time, and powers and products of trigonometric functions arising from substitution into the differential equation are re-expressed in terms of linear combinations of multiple-angle functions. Then higher-multiple functions are discarded, and coefficients of the lowest-order terms are matched ("balanced"). Interest in "jerk" equations

$$
\dddot{\mathrm{x}}=\mathrm{J}(\mathrm{x}, \dot{\mathrm{x}}, \ddot{\mathrm{x}})
$$

involving the third temporal derivative of displacement (which might also be termed “triceleration"), has recently been rekindled [2]. As well as originally being of some interest in mechanics (see e.g. refs [3], [4]), nonlinear jerk equations are finding increasing importance in the study of chaos (see the article by von Baeyer [5], and the recent paper by Sprott \& Linz [6] and references therein). Many third-order nonlinear systems (three simultaneous first-order nonlinear differential equations), both mathematically and physically motivated, such as the now-classical Rössler system [7], may be recast into a single nonlinear third-order differential (jerk) equation involving only one of the dependent variables by suitable elimination [2], [8]. Some early investigations into nonlinear jerk equations (although not termed as such) 
include oscillations in a nonlinear vacuum tube circuit [9], and third-order mechanical oscillators [10], [11], [12]. Other physical situations in which nonlinear jerk-type equations have been investigated, with more emphasis on chaotic solutions (called aperiodic in earlier works), include a thermo-mechanical oscillator model with thermal dissipation [13], [14], fluid dynamical convection [15], and stellar ionization zone oscillations [16]. Jerk equations, though not nearly as common as acceleration (or force) equations $\ddot{\mathrm{x}}=\mathrm{f}(\mathrm{x}, \dot{\mathrm{x}})$, are therefore of direct physical interest. Moreover, simple forms of the jerk function $\mathrm{J}$ which lead to perhaps the simplest manifestation of chaos have been found by Sprott [17].

This paper sets out to investigate not chaotic solutions to jerk equations (as many of the above references do), but the analytical approximations, via the method of harmonic balance, to periodic solutions to nonlinear jerk equations in appropriate parameter regions where these exist. Such solutions do not appear to have been dealt with by other authors before. At first sight, difficulties seem apparent in this approach. First of all, a couple of facts contributing to the usefulness of the HB method in investigating nonlinear second-order differential (acceleration) equations are mentioned. (i) The linear d.e. $\ddot{x}=-\omega^{2} x$ has two independent periodic solutions $\{\cos ; \sin \}(\omega t)$. (ii) Two initial conditions, on $\mathrm{x}$ and its derivative, are required, and it is always possible to have only one of these, viz. $\cos (\omega t)$, non-zero at $\mathrm{t}=0$.

On the other hand, for third-order differential (jerk) equations, the situation is different. (i) The linear d.e. $\dddot{x}=-\omega^{3} x$ does not have any periodic solutions. (The special linear third-order o.d.e. $\dddot{x}+\omega^{2} \dot{x}+\alpha\left(\ddot{x}+\omega^{2} x\right)=0$ does possess the above two independent periodic trigonometric solutions, but also (for $\alpha \neq 0$ ) may have a third, nonperiodic, solution.) (ii) The third-order d.e. requires three initial conditions, for $\mathrm{x}$ 
and its first and second derivatives. Assuming an approximate solution to a third-order nonlinear d.e. of the form $\mathrm{x} \sim \cos (\Omega \mathrm{t})$, it is not possible to have only one non-zero initial condition at $\mathrm{t}=0$, because $\ddot{\mathrm{x}}(0)$ is also required which moreover would involve the unknown $\Omega$.

Difficulty (i) can be overcome by considering jerk equations whose linear term, if any, involves only $\dot{\mathrm{x}}$. Difficulty (ii) may be addressed by using the Ansatz $\mathrm{x} \sim$ $\sin (\Omega t)$, so that only the initial velocity is non-zero, with the initial displacement and also acceleration being zero. A consequent restriction on the jerk equations amenable to harmonic balance using this approach is that (choosing time $\mathrm{t}=0$ when $\mathrm{x}=0$ ) only problems with zero initial acceleration can be considered. This has to be appreciated from the outset. However, this situation of starting off with a constant velocity is a feasible condition, depending on the actual physical meaning of the dependent variable $\mathrm{x}$ and interpretation of the equation. For instance, the initial conditions $(x(0)=0, \dot{x}(0) \neq 0, \ddot{x}(0)=0)$ are quite common in investigations of third-order d.e.s, such as for example those corresponding to physically implementable chaotic electrical circuits [18]. 


\section{SUITABLE JERK FUNCTIONS}

It is well known from the situation for second-order d.e.s described in the first paragraph of the Introduction that the harmonic balance method for finding periodic solutions works most successfully for equations which are parity-invariant and timereversal invariant, i.e. all terms have the same space-parity (behaviour under $\mathrm{x} \rightarrow-\mathrm{x}$ ) and time-parity (behaviour under $t \rightarrow-t$ ). For d.e.s involving the jerk $\dddot{x}=J(x, \dot{x}, \ddot{x})$ there should therefore only be odd powers of the x-type variables and only an odd total number of time derivatives in each term. Thus equations such as the equation (30) cited at the end of a recent paper by Mickens [19], containing an $x^{3}$ nonlinearity, do not fall within the ambit of the present paper. Further, the only linear term of the three possible arguments is $\dot{\mathrm{x}}$. Thus jerk equations containing a term linear in $\ddot{\mathrm{x}}$, such as in references [9-16] discussed above, are not considered here. There are ten possible cubic-type terms (for simplicity in this paper, we do not go higher than thirdorder nonlinearities), of which only the following four meet the requirements as desired: (I) $\mathrm{x}^{2} \dot{\mathrm{x}}$; (II) $\dot{\mathrm{x}}^{3}$; (III) $\mathrm{x} \dot{\mathrm{x}} \ddot{\mathrm{x}}$; (IV) $\dot{\mathrm{x}}^{2}$. The harmonic balance approach to such third-order nonlinear differential equations does not appear to have been carried out before.

Interest here is in distinct jerk equations, not merely equivalent to or derived from second-order equations. Thus first of all the linear jerk equation $\dddot{\mathrm{X}}=-\dot{\mathrm{x}}$ is simply the time derivative of a linear acceleration equation, in the form $(\mathrm{d} / \mathrm{dt})[\ddot{\mathrm{x}}]=(\mathrm{d} / \mathrm{dt})[-\mathrm{x}]$; and the third solution is a constant. The third-order equation corresponding to (I) is $\dddot{x}=-x^{2} \dot{x}$ which is simply the time-derivative of an 
acceleration equation, when written as $(\mathrm{d} / \mathrm{dt})[\ddot{\mathrm{x}}]=(\mathrm{d} / \mathrm{dt})\left[-(1 / 3) \mathrm{x}^{3}\right]$, and so is not regarded as distinctively jerk-like. The third-order equation corresponding to (II) is $\dddot{x}=-\dot{x}^{3}$, which is equivalent to the second-order system $\ddot{y}=-y^{3}$ with $y=\dot{x} \cdot$ (This equation, termed the "velocillator", was analyzed in some detail in [20].) Similar statements may be made concerning the preceding two nonlinear d.e.s if a linear term involving $\dot{\mathrm{x}}=(\mathrm{d} / \mathrm{dt})(\mathrm{x})=\mathrm{y}$ is added. The other two allowed nonlinear forms (III) and (IV) listed in the preceding paragraph involve the higher, second-order, derivative $\ddot{\mathrm{x}}$, and will be considered later. 


\section{GENERAL NONLINEAR JERK FUNCTIONS}

The most general jerk function which is invariant under time-reversal and space-reversal and which has only cubic nonlinearities as specified in Section 2 above may be written as

$$
\dddot{x}=-\gamma \dot{\mathrm{x}}-\alpha \dot{\mathrm{x}}^{3}-\beta \mathrm{x}^{2} \dot{\mathrm{x}}+\delta \mathrm{x} \dot{\mathrm{x}} \ddot{\mathrm{x}}-\varepsilon \dot{\mathrm{x}} \ddot{\mathrm{x}}^{2},
$$

where the parameters $\gamma, \alpha, \beta, \delta$ and $\varepsilon$ are constants (and the signs have been chosen to most naturally suit the harmonic balance procedures). By rescaling $\mathrm{x}$ and/or $\mathrm{t}$, any one or two of these parameters (except $\alpha$ and $\delta$ simultaneously) may be set to unity to obtain standard equations with fewer control parameters suitable for more extensive study. (If the behaviour as the coefficient of a particular term becomes very small is to be investigated, that parameter should not of course be thus "normalized". )

As mentioned above, this paper is not concerned with jerk equations which may simply be recast into a second-order equation for the variable $y=\dot{x}$, so at least one of $\beta, \delta, \varepsilon$ should be nonzero. Furthermore, as also mentioned in Section 2 above, we are not here interested in jerk equations which are simply the time-derivative of an acceleration equation. Such equations, which may be termed "Newtonian jerk" (c.f. [8], [21]), have the form $\dddot{x}=(\partial f / \partial x) \dot{x}+(\partial f / \partial \dot{x}) \ddot{x}$ where $\ddot{x}=f(x, \dot{x})$ can be considered as an expression of Newton's Second Law for $\mathrm{f}$, the force function per unit mass. Thus, if $\varepsilon=0$, we would require $\delta \neq-2 \alpha\left(\right.$ since $\left.\dot{x}^{3}+2 x \dot{x} \ddot{x}=(d / d t)\left[x \dot{x}^{2}\right]\right)$.

Substitution, according to the Introduction, of $\mathrm{x}(\mathrm{t})=(\mathrm{B} / \Omega) \sin (\Omega \mathrm{t})$ into equation (3.1), and use of the harmonic balance procedure, leads, from the coefficient of $\cos (\Omega t)$, to 


$$
\left[4-\varepsilon \mathrm{B}^{2}\right] \Omega^{4}-\left[4 \gamma+(3 \alpha+\delta) \mathrm{B}^{2}\right] \Omega^{2}-\beta \mathrm{B}^{2}=0 .
$$

This quadratic equation in $\Omega^{2}$ may be solved to give positive $\Omega$ in terms of the initial velocity amplitude B as the harmonic balance approximation to the angular frequency and thence the period. If also the coefficient of the $\cos (3 \Omega \mathrm{t})$ term in the HB procedure is zero, viz.

$$
\varepsilon \Omega^{4}+(\delta-\alpha) \Omega^{2}+\beta=0
$$

then the above sine solution for $\mathrm{x}(\mathrm{t})$ is will be exact; but this will only sometimes be possible.

The general equation (3.1) and the approximate frequency equation (3.2) involving three essential control parameters and one amplitude parameter are not easily analyzable in generality as regards their key features, and will not as such be pursued further here. Rather, several special cases of equation (3.1) will be dealt with in some explicit detail in succeeding Sections. 


\section{JERK FUNCTION CONTAINING VELOCITY-CUBED AND VELOCITY TIMES DISPLACEMENT-SQUARED}

We now note that the simplest, parity- and time-reversal-invariant, distinctive jerk equation with polynomial nonlinearities and lowest time-derivatives will involve a linear combination of the cubic forms (I) and (II) above. This was analyzed as a paradigm in [20], where it was shown that all of its solutions are periodic and phase plane curves (for initial condition $\dot{\mathrm{x}}(0)=0$ ) were found; that work was not concerned with the actual values of the periods. In this section, as a first detailed application of the harmonic balance method to a jerk equation, approximations to the periods of this paradigm equation are derived, and compared with exact results found by numerical integration of the third-order d.e..

The equation to be investigated is

$$
\dddot{\mathrm{x}}=-\dot{\mathrm{x}}\left(\dot{\mathrm{x}}^{2}+\mathrm{x}^{2}\right)
$$

Without loss of generality, given that both terms appear on the right hand side, the coefficients of both these terms can always be set to 1 by appropriate rescalings of displacement $\mathrm{x}$ and time $\mathrm{t}$. The harmonic balance approximation for such equations is

$$
\mathrm{x}=(\mathrm{B} / \Omega) \sin (\Omega \mathrm{t}) \text {, with } \mathrm{x}(0)=0,
$$

so

$$
\begin{aligned}
& \dot{\mathrm{x}}=\mathrm{B} \cos (\Omega \mathrm{t}), \text { with } \dot{\mathrm{x}}(0)=\mathrm{B} ; \\
& \ddot{\mathrm{x}}=-\Omega \mathrm{B} \sin (\Omega \mathrm{t}), \text { with } \ddot{\mathrm{x}}(0)=0,
\end{aligned}
$$

where $\Omega$ is the angular frequency. Then the period is given by

$$
\mathrm{T}=2 \pi / \Omega
$$

and the (approximate) displacement amplitude in equation (4.2a) is given by 


$$
\mathrm{A}=\mathrm{B} / \Omega \text {. }
$$

Note that the above choice of the form (4.2a) of $x$ allows for the non-zero initial condition, for $\dot{\mathrm{x}}$, to involve the independent velocity-amplitude constant $\mathrm{B}$, which may in principle be assigned arbitrarily. The forms for $\mathrm{x}$ and $\ddot{\mathrm{x}}$ involve the unknown frequency parameter $\Omega$, but this does not appear in their initial conditions because they are zero. As mentioned in the Introduction, for the harmonic balance method to be applicable as formulated here, the initial acceleration, as well as the initial displacement, should be zero.

Substitution into equation (4.1), and manipulation using trigonometric identities, yields, for $\mathrm{B} \neq 0$,

$$
\begin{aligned}
\Omega^{2} \cos (\Omega \mathrm{t})= & (1 / 4)\left(\mathrm{B}^{2} / \Omega^{2}\right)\left(3 \Omega^{2}+1\right) \cos (\Omega \mathrm{t}) \\
+ & (1 / 4)\left(\mathrm{B}^{2} / \Omega^{2}\right)\left(\Omega^{2}-1\right) \cos (3 \Omega \mathrm{t}) .
\end{aligned}
$$

It is immediately apparent that if $\mathrm{B}=1$ and $\Omega=1$ then equation (4.7) is satisfied exactly: $\mathrm{x}=\sin (\mathrm{t})$ is an exact solution of equation (4.1), as can easily be verified by direct substitution. If $\mathrm{B} \neq 1$, then the harmonic balance approximation is invoked: the higher harmonic in equation (4.7) is ignored and the coefficients of $\cos (\Omega \mathrm{t})$ are equated, yielding $\mathrm{B}^{2}=4 \Omega^{4} /\left(3 \Omega^{2}+1\right)$ and thence the approximate expression for angular frequency $\Omega(>0)$ in terms of velocity amplitude B:

$$
\Omega=[1 /(2 \sqrt{2})] \sqrt{3 B^{2}+\sqrt{9 B^{4}+16 B^{2}}} .
$$

It can be seen that, in this approximation, as $\mathrm{B} \rightarrow 0, \Omega \rightarrow 0$, i.e. $\mathrm{T} \rightarrow \infty$, and $\mathrm{A} \sim$ $\sqrt{ } 2 \sqrt{ } \mathrm{B} \rightarrow 0$. As $\mathrm{B} \rightarrow \infty, \mathrm{T} \rightarrow 0$, i.e. $\Omega \rightarrow \infty$, and $\mathrm{A} \rightarrow 2 / \sqrt{ } 3=1.15470054$

The numerical values for period $\mathrm{T}$ (4.5) and also the displacement amplitude A (4.6) resulting from the approximate expression (4.8), for a range of values of initial velocity amplitude B, are compared in Table 1 with the exact numerical values 
obtained by solving the third-order differential equation (4.1) with initial conditions (4.2b), (4.3b), (4.4b) using the computational software ODE Workbench [22]. For these, as for subsequent, computations, it was checked that the phase plane ( $\dot{\mathrm{x}} v s \mathrm{x}$ ) orbits did indeed close. Furthermore, since these are third-order d.e.s, it was also checked, here and subsequently, that the orbits in another phase plane ( $\dot{\mathrm{x}} v s \ddot{\mathrm{x}})$ were also closed and resulted in the same computed period. (Whilst orbits in the first case progressed clockwise, those in the second case progressed anti-clockwise. Note that in the present instance, the initial conditions in both phase planes are the same.)

It may be mentioned that for equation (4.1) the exact phase plane orbits were given in reference [20]. For the present initial conditions (4.2b), (4.3b), (4.4b) they are given by

$$
\dot{\mathrm{x}}^{2}+\mathrm{x}^{2}=1+\left(\mathrm{B}^{2}-1\right) \cos (\sqrt{2} \mathrm{x})
$$

and they form a set of nested, closed curves. Successive differentiations of equation (4.9) show that the effective potential there has a minimum at $x=0$, generating a stable centre. The associated closed paths (4.9) therefore are Poincare stable orbits (see, for example, the text-book by Jordan and Smith [23, p.280].)

The exact displacement amplitude defined as the value of $\mathrm{x}$ when $\dot{\mathrm{x}}=0$ satisfies the equation, following from (4.9),

$$
\mathrm{A}^{2}-1+\left(1-\mathrm{B}^{2}\right) \cos (\sqrt{2} \mathrm{~A})=0,
$$

so for given B the exact value for A can be found by numerical solution of this algebraic equation. The results agree with the results obtained by numerical solution of the differential equation, thus checking the accuracy of the ODE software [22].

The accuracy of the harmonic balance approximation as exhibited in Table 1 is very good throughout the wide range of $\mathrm{B}$, being better than about $7 \%$ for the period. (The "exact" results for the $B=1$ case corresponding to the exact solution $x=\sin (t)$ 
with $\mathrm{T}=2 \pi$ and $\mathrm{A}=1$ are quoted to more significant figures to further confirm the accuracy of the numerical software.) The accuracy is better for B values near $B=1$, and worsens a little as B departs from this value. The reason for this may be understood by making the next harmonic balance approximation, as outlined in the following sub-section.

\subsection{HARMONIC-BALANCE CORRECTION}

The correction to the simple harmonic balance approximation is obtained in a way similar to that for the more usual second-order nonlinear oscillator equations [1] by adding a third-harmonic term, for convenience in the present case as follows:

$$
\mathrm{x}=(\mathrm{B} / \Omega) \sin (\Omega \mathrm{t})+(\mathrm{b} /(3 \Omega)) \sin (3 \Omega \mathrm{t}),
$$

so that the initial velocity is now given by $\dot{\mathrm{x}}(0)=\mathrm{B}+\mathrm{b}$. Two equations are now obtained by equating the coefficients of $\cos (\Omega t)$ and of $\cos (3 \Omega t)$. After some manipulation there results the ratio

$$
\mathrm{b} / \mathrm{B}=(1 / 7)\left(\Omega^{2}-1\right) /\left(3 \Omega^{2}+1\right) \quad .
$$

(This vanishes when $\Omega=1$, corresponding to the exact solution where $b=0$.) Since the right side of equation (4.12) is an increasing function of $\Omega^{2}$, it follows that

$$
-1 / 21<\mathrm{b} /(3 \mathrm{~B})<1 / 63 \text { for } 0<\Omega^{2}<\infty \text {, }
$$

so the ratio of coefficients in the expression (4.11) for $\mathrm{x}$ has modulus always less than $1 / 21$, i.e. the amplitude correction is less than $5 \%$, which is small as desired.

Furthermore, from equation (4.13), better relative accuracy is expected for $\Omega$ large than for small, i.e. for T small rather than large. This is borne out in Table 1. 


\subsection{JERK FUNCTION CONTAINING VELOCITY-CUBED,}

\section{VELOCITY TIMES DISPLACEMENT-SQUARED, AND VELOCITY}

This section investigates a slightly more complicated case which extends the jerk function of the above section by incorporating a linear, velocity, term. The equation is first presented with general coefficients:

$$
\dddot{x}=-\dot{x}\left(\alpha \dot{x}^{2}+\beta x^{2}\right)-\gamma \dot{x}
$$

Since the $\beta=0$ case of this equation is just a second-order d.e. for the dependent variable $y=\dot{x}$, and the $\alpha=0$ case is just the time-derivative of the acceleration equation $\ddot{\mathrm{x}}=-\beta \mathrm{x}^{3} / 3-\gamma \mathrm{x}$, both the cubic terms are needed for a non-trivial jerk equation. By rescaling, both coefficients $\alpha$ and $\beta$ may be set equal to unity as before, and $\gamma$ remains the single essential control parameter.

Two identities involving jerk were introduced in reference [20]:

$$
\begin{aligned}
\dddot{x} & \equiv \frac{1}{2} \dot{\mathrm{x}} \frac{\mathrm{d}^{2}}{\mathrm{dx}^{2}}\left(\dot{\mathrm{x}}^{2}\right) \\
& \equiv \dot{\mathrm{x}} \frac{\mathrm{d} \ddot{\mathrm{x}}}{\mathrm{dx}}
\end{aligned}
$$

Use of the first identity (4.15a) enables one to find the explicit phase plane curve equations for the d.e. (4.14) (with $\alpha=\beta=1$ ), for the initial conditions of this paper:

$$
\dot{\mathrm{x}}^{2}+\mathrm{x}^{2}=1-\gamma+\left(\mathrm{B}^{2}-1+\gamma\right) \cos (\sqrt{2} \mathrm{x})
$$

These are again closed, nested curves, with the $\gamma$ term in equation (4.14) just modifying constants in equation (4.16); this may be compared with equation (4.9) above for the case $\gamma=0$, so the orbits are again stable (c.f. Sect. 4 . above).

The harmonic balance approach via equations (4.2) - (4.4) is now applied to the rescaled jerk equation 


$$
\dddot{\mathrm{x}}=-\dot{\mathrm{x}}\left(\dot{\mathrm{x}}^{2}+\mathrm{x}^{2}\right)-\gamma \dot{\mathrm{x}} \quad \text {. }
$$

The coefficient of $\cos (\Omega t)$ yields the relation

$$
\mathrm{B}^{2}=4 \Omega^{2}\left(\Omega^{2}-\gamma\right) /\left(3 \Omega^{2}+1\right) .
$$

From the coefficient of the $\cos (3 \Omega \mathrm{t})$ term, it is found that the solution is exact if $\Omega=$ 1, i.e. $B=\sqrt{1-\gamma}$. Thus no exact sine solution is possible if $\gamma>1$. If $B \neq \sqrt{1-\gamma}$, then equation (4.18) may be solved to find the approximate harmonic balance expression for the angular frequency in terms of the initial velocity amplitude:

$$
\Omega=[1 /(2 \sqrt{2})] \sqrt{3 B^{2}+4 \gamma+\sqrt{\left(3 B^{2}+4 \gamma\right)^{2}+16 B^{2}}} .
$$

Two examples are considered, first for parameter value $\gamma=1 / 2$, for which the possibility of an exact solution exists for suitable choice of $B(=1 / \sqrt{2}$ in this case), and secondly for $\gamma=2$, for which it was found above that no exact sinusoidal solution exists. Some representative results are presented in Tables 2(a),(b). The harmonic balance results for period $\mathrm{T}$ and displacement amplitude A are remarkably accurate, within a couple of per cent, even in case (b) where no exact sine solution is possible. 


\section{JERK FUNCTIONS CONTAINING THE ACCELERATION}

The functions (III) and (IV) listed in Section 2 above involve the second-order time-derivative, as distinct from the functions dealt with in Section 4 . They will be now be dealt with in turn.

\subsection{JERK FUNCTION CONTAINING DISPLACEMENT TIMES VELOCITY TIMES ACCELERATION, AND VELOCITY}

For the function (III), after rescaling of the x variable, the corresponding jerk equation would take the form

$$
\dddot{\mathrm{x}}=\mathrm{x} \dot{\mathrm{x}} \ddot{\mathrm{x}} \text {. }
$$

The harmonic balance approximation would give $\Omega=\mathrm{B} / 2$ so $\mathrm{T}=4 \pi / \mathrm{B}$ and $\mathrm{A}=2$.

However, the solution $\dot{\mathrm{x}}=\mathrm{B}=$ constant to equation (5.1) satisfies the initial conditions $(4.3 \mathrm{~b}),(4.4 \mathrm{~b})$, and this is the solution obtained by numerical integration. The solution is not periodic, and the harmonic balance approach is spurious in this

instance. From the identity (4.15b) together with $\ddot{x}=(d / d x)\left(\dot{x}^{2} / 2\right)$ follow the phase plane equations

$$
\dot{\mathrm{x}}^{2}+\mathrm{c}_{1} \int_{0}^{\mathrm{x}} \exp \left(\frac{1}{2} \mathrm{X}^{2}\right) \mathrm{dX}=\mathrm{B}^{2},
$$

where $\mathrm{c}_{1}$ is some constant, satisfying the initial conditions $\mathrm{x}(0)=0 ; \dot{\mathrm{x}}(0)=\mathrm{B}$. For $\mathrm{c}_{1}=0$, the above constant velocity solution is obtained. If $\mathrm{c}_{1} \neq 0$, the curves (5.2) are not closed. 
To find an equation amenable to harmonic balance analysis, a term linear in the velocity is incorporated. The resulting standardized jerk equation, after rescaling of both $\mathrm{x}$ and $\mathrm{t}$, is therefore taken to be

$$
\dddot{\mathrm{x}}=\mathrm{x} \dot{\mathrm{x}} \ddot{\mathrm{x}}-\dot{\mathrm{x}}
$$

(which is free of parameters). The phase plane curves for this equation may be written in the form

$$
\dot{\mathrm{x}}^{2}+\mathrm{c}_{1} \int_{0}^{\mathrm{X}} \exp \left(\frac{1}{2} \mathrm{X}^{2}\right) \mathrm{dX}+2 \int_{0}^{\mathrm{X}} \exp \left(\frac{1}{2} \mathrm{X}^{2}\right)\left[\int_{0}^{\mathrm{X}} \exp \left(-\frac{1}{2} \xi^{2}\right) \mathrm{d} \xi\right] \mathrm{dX}=\mathrm{B}^{2}
$$

Harmonic balance now gives the approximate result

$$
\Omega=(1 / 2) \sqrt{\mathrm{B}^{2}+4}
$$

with period T and displacement amplitude A then given by (4.5) and (4.6). (There is no exact sinusoidal solution for any value of B.) Table 3 gives some results, compared with exact computed results, for a range of values of $\mathrm{B}$, over which the phase plane orbits form nested closed curves and hence are again stable. Note that, in this case, as $\mathrm{B} \rightarrow 0, \mathrm{~T} \rightarrow 2 \pi$ (corresponding to a small amplitude linear oscillator), and this is reflected in the trend. The harmonic balance results are very good, especially for the period, improving as B decreases.

To justify the approach and explain the good results for $\mathrm{B} \lesssim 1$, the harmonic balance correction (4.11) may here be inserted into equation (5.3). It is found that, to first order in $\mathrm{b} / \mathrm{B}$, this ratio is approximately given by

$$
\mathrm{b} / \mathrm{B} \approx 1 /\left[\left(32 / \mathrm{B}^{2}\right)-7\right] \text {. }
$$

If $B \leq 1$, the ratio of coefficients $b /(3 B)$ in (4.11) for this equation is therefore less than $1 / 75 \approx 1.3 \%$ which is indeed small. 


\subsection{JERK FUNCTION CONTAINING VELOCITY TIMES}

\section{ACCELERATION-SQUARED, AND VELOCITY}

For the final function (IV) of Section 2, after rescaling of the $\mathrm{x}$ variable, the corresponding jerk equation may be taken to have the form

$$
\dddot{\mathrm{x}}=-\dot{\mathrm{x}} \ddot{\mathrm{x}}^{2}
$$

The harmonic balance approximation would actually give $\mathrm{B}=2$, with no value for $\Omega$.

However, once again the solution $\dot{\mathrm{x}}=\mathrm{B}=$ constant is a solution to equation (5.7)

which satisfies the initial conditions (4.3b), (4.4b), and this is again the solution obtained by numerical integration. The harmonic balance approach is again spurious in this instance. From the identity (4.15b) follow the phase plane equations

$$
\frac{1}{2} \dot{x}^{2}-\ln \left[\left|x+c_{1}\right|\right]=c_{2}
$$

where $c_{1}$ and $c_{2}$ are constants. These curves are not closed.

To find an equation amenable to harmonic balance analysis, a term linear in the velocity is again incorporated. The resulting jerk equation is written in the form which for the present retains the parameter $\gamma>0$ :

$$
\dddot{\mathrm{x}}=-\dot{\mathrm{x}} \ddot{\mathrm{x}}^{2}-\gamma \dot{\mathrm{x}}
$$

The phase plane curves may be written in the form

$$
\frac{1}{2} \dot{\mathrm{x}}^{2}-\ln \left[\left|\cos \left(\sqrt{\gamma} \mathrm{x}+\mathrm{C}_{1}\right)\right|\right]=\mathrm{C}_{2},
$$

where $\mathrm{C}_{1}$ and $\mathrm{C}_{2}$ are constants.

Harmonic balance gives the approximate equation

$$
\Omega=2 \sqrt{\gamma /\left(4-\mathrm{B}^{2}\right)},
$$


for $\mathrm{B}<2$. Results have been given so far with parameter $\gamma$ included because, for instance, the form of equation (5.8) cannot immediately be deduced from equation (5.10) by letting $\gamma \rightarrow 0$, although compatibility can be achieved by a careful consideration of the relations between the constants specified in the phase plane equations. (See Appendix A.)

Now the jerk equation may be brought into a standard form by also rescaling the time to give

$$
\dddot{\mathrm{x}}=-\dot{\mathrm{x}} \ddot{\mathrm{x}}^{2}-\dot{\mathrm{x}} \text {. }
$$

The expression (5.11) becomes

$$
\Omega=2 / \sqrt{4-B^{2}},
$$

and the period $\mathrm{T}$ and the displacement amplitude $\mathrm{A}$ are then given by (4.5) and (4.6). (Here again there is no exact sinusoidal solution for any value of B.) Table 4 gives some results, compared with exact computed results, for a range of values of B. Note that, as $\mathrm{B} \rightarrow 0$, again $\mathrm{T} \rightarrow 2 \pi$, and this is reflected in the trend. The harmonic balance results are very good, particularly for $\mathrm{B}<1$. (Evidently B cannot get too large for the approximation (5.13) to be relevant.)

It may be noted that the exact phase plane curves for the jerk equation (5.12), with the present initial conditions, may be written as

$$
\frac{1}{2} \dot{\mathrm{x}}^{2}+\log _{\mathrm{e}}[|\sec (\mathrm{x})|]=\frac{1}{2} \mathrm{~B}^{2} .
$$

For oscillations about $\mathrm{x}=0$, the second term can be interpreted as a potential whose shape guarantees stability of the orbits. Furthermore, in this case the exact displacement amplitude may be found explicitly by the expression

$$
A=\operatorname{arcos}\left(\exp \left(-B^{2} / 2\right)\right)
$$

This checks the ODE numerical solution results for A in Table 4. 


\section{DISCUSSION}

In this paper, parity- and time-reversal- invariant jerk equations with cubic nonlinearities were subjected to a harmonic balance analysis. The results for periods and displacement amplitudes for a number of different types of appropriate equations were compared with the results of numerical computations and found to be generally in good agreement over a range of the initial velocity amplitude values. Evidently the work could be extended to higher order, in particular quintic, nonlinearities.

Mixed-parity equations were avoided, as they are known to produce difficulties for the harmonic balance method even for second-order "acceleration" nonlinear equations [24], [25]. However, they may be important, because the "simplest" chaotic third-order d.e.s [17] involve only quadratic nonlinearities, such as $\mathrm{x} \dot{\mathrm{x}}, \dot{\mathrm{x}}^{2}$, or $\mathrm{x}^{2}$ (although the latter two have the opposite time-reversal behaviour).

The condition $\ddot{x}=0$ utilized above, whilst not unusual, is certainly restrictive. This raises the question as to whether some other set of periodic trial functions, rather than the two standard trigonometric functions, may be more suitable for third-order nonlinear equations. For instance, the three Jacobian elliptic functions sn, cn, dn [26] have found some successful applications for second-order nonlinear d.e.s [27], [28]. (In particular, their derivatives [26] involve the other two of the trio, as compared with the other one for the pair sin, cos.) 


\section{ACKNOWLEDGMENTS}

The author thanks the Department of Mathematics at the University of Queensland for hospitality during an Academic Studies Program visit there. Conversations with Drs Tony O'Connor and Peter Johnston at Griffith University were appreciated. 


\section{REFERENCES}

1. R. E. Mickens 1996 Oscillations in Planar Dynamical Systems. Singapore: World Scientific. Chapter 4.

2. H. P. W. Gottlieb 1996 American Journal of Physics 64, 525. Question \#38. What is the simplest jerk function that gives chaos?

3. S. H. Schot 1978 American Journal of Physics 46, 1090-1094. Jerk: The time rate of change of acceleration.

4. T. R. Sandlin 1990 The Physics Teacher 28, 36-40. The jerk.

5. H. C. von Baeyer 1998 The Sciences 38, 12-14. All shook up.

6. J. C. Sprott and S. J. Linz 2000 International Journal of Chaos Theory and Applications 5, 3-22. Algebraically simple chaotic flows.

7. O. E. Rössler 1976 Physics Letters A 57, 397-398. An equation for continuous chaos.

8. S. J. Linz 1997 American Journal of Physics 65, 523-526. Nonlinear dynamical models and jerky motion.

9. L. L. Rauch 1950 Annals of Mathematics Studies 20, 39-88 . Oscillation of a third order nonlinear autonomous system. (In: S. Lefschetz (Ed.) Contributions to the Theory of Nonlinear Oscillations. Princeton: Princeton University Press.)

10. B. V. Dasarathy and P. Srinivasan 1969 Journal of Sound and Vibration 9, 49-52. On the study of a third-order mechanical oscillator.

11. H. R. Srirangarajan, P. Srinivasan and B.V. Dasarathy 1975 Journal of Sound and Vibration 40, 162-172. Ultraspherical polynomials approach to the study of thirdorder non-linear systems. 
12. H. R. Srirangarajan and B.V. Dasarathy 1975 Journal of Sound and Vibration 40, 173-178. Study of third-order non-linear systems - variation of parameters approach.

13. D. W. Moore and E. A. Spiegel 1966 The Astrophysical Journal 143, 871-887. A thermally excited non-linear oscillator.

14. N. H. Baker, D. W. Moore and E. A. Spiegel 1971 Quarterly Journal of Mechanics and Applied Mathematics 24, 391-422. Aperiodic behaviour of a nonlinear oscillator.

15. A. Arneodo, P. H. Coullet and E. A. Spiegel 1982 Physics Letters 92A, 369-373. Chaos in a finite macroscopic system.

16. M. Auvergne and A. Baglin 1985 Astronomy and Astrophysics 142, 388-392. A dynamical instability as a driving mechanism for stellar oscillations.

17. J. C. Sprott 1997 Physics Letters A 228, 271-274. Simplest dissipative chaotic flow.

18. J. C. Sprott 2000 American Journal of Physics 68, 758-763. Simple chaotic systems and circuits.

19. R.E. Mickens 2002 Journal of Sound and Vibration 255, 789-792. Analysis of non-linear oscillators having non-polynomial elastic terms.

20. H. P. W. Gottlieb 1998 American Journal of Physics 66, 903-906. Simple nonlinear jerk functions with periodic solutions.

21. S. J. Linz 1998 American Journal of Physics 66, 1109-1114. Newtonian jerky dynamics: Some general properties.

22. J.M. Aguirregabiria 1994 ODE Workbench. New York: American Institute of Physics. (PC Version 1.5) 
23. D.W. Jordan and P.Smith 1999 Nonlinear ordinary differential equations. 3rd edition. Oxford: Oxford University Press.

24. A. H. Nayfeh and D. T. Mook 1979 Nonlinear Oscillations. New York: John Wiley. Section 2.3.4.

25. H. P. W. Gottlieb 1992 Journal of Sound and Vibration 152, 189-191. On the harmonic balance method for mixed-parity non-linear oscillators.

26. D. F. Lawden 1989 Elliptic Functions and Applications. New York: Springer. Chapter 2.

27. S. Bravo Yuste 1992 International Journal of Non-linear Mechanics 27, 347-356. "Cubication" of non-linear oscillators using the principle of harmonic balance.

28. M. Belhaq and F. Lakrad 2000 Journal of Sound and Vibration 233, 935-937. On the elliptic harmonic balance method for mixed parity non-linear oscillators. 
TABLE 1

Values for period $T$ and displacement amplitude A of equation (4.1) for initial conditions (4.2b, 4.3b, 4.4b) as given by formulae (4.5) and (4.6) with harmonic balance (harm. bal.) equation (4.8), for a range of values of initial velocity amplitude $B$, together with exact values obtained by numerical computation. (For the $B=1$ case, harmonic balance is exact.)

\begin{tabular}{lllll} 
& \multicolumn{2}{c}{ T } & & \\
B & harm. bal. & exact & harm. bal. & exact \\
\hline .1 & 27.065998 & 25.359725 & 0.430769 & 0.468121 \\
.2 & 18.438632 & 17.495410 & 0.586920 & 0.627919 \\
.5 & 10.461083 & 10.210761 & 0.832466 & 0.860011 \\
1 & $2 \pi=6.283185307$ & 6.283185307 & 1 & 1.000000000 \\
2 & 3.457326 & 3.508793 & 1.100501 & 1.074352 \\
5 & 1.438527 & 1.468638 & 1.144743 & 1.104257 \\
10 & 0.723920 & 0.739762 & 1.152154 & 1.109078 \\
20 & 0.362559 & 0.370580 & 1.154060 & 1.110308 \\
\hline
\end{tabular}


TABLE 2

Values for period $T$ and displacement amplitude A of equation (4.17) for initial conditions $(4.2 b, 4.3 b, 4.4 b)$ as given by formulae (4.5) and (4.6) with harmonic balance (harm. bal.) equation (4.19), for a range of values of initial velocity amplitude B, together with exact values obtained by numerical computation, for two values of the parameter $\gamma$. (For the $\gamma=1 / 2$ case with $B=1 / \sqrt{ } 2$, harmonic balance is exact.)

(a) $\gamma=1 / 2$

$\mathrm{T}$

B harm. bal. exact harm. bal. exact

\begin{tabular}{lllll}
\hline .5 & 7.165959 & 7.160833 & 0.570249 & 0.572279 \\
$1 / \sqrt{ } 2$ & $2 \pi=6.283185307$ & 6.283185307 & $1 / \sqrt{ } 2=0.7071067812$ & 0.7071067812 \\
1 & 5.262752 & 5.276160 & 0.837593 & 0.831912 \\
2 & 3.237910 & 3.276060 & 1.030659 & 1.006639 \\
\hline
\end{tabular}


[Table 2, continued]

(b) $\gamma=2$

$\mathrm{T}$

\begin{tabular}{lllll} 
B & harm. bal. & exact & harm. bal. & exact \\
\hline .5 & 4.221081 & 4.221545 & 0.335903 & 0.335066 \\
1 & 3.729641 & 3.734078 & 0.593591 & 0.588522 \\
2 & 2.757326 & 2.775201 & 0.877684 & 0.859563 \\
\hline
\end{tabular}

A 
TABLE 3

Values for period $T$ and displacement amplitude A of equation (5.3) for initial conditions $(4.2 b, 4.3 b, 4.4 b)$ as given by formulae (4.5) and (4.6) with harmonic balance (harm. bal.) equation (5.5), for a range of values of initial velocity amplitude $B$, together with exact values obtained by numerical computation.

A

B harm. bal. exact harm. bal. exact

\begin{tabular}{lllll}
\hline .1 & 6.275346 & 6.275347 & 0.099875 & 0.099917 \\
.2 & 6.252003 & 6.252016 & 0.199007 & 0.199338 \\
.5 & 6.095585 & 6.096061 & 0.485071 & 0.489978 \\
1 & 5.619852 & 5.626007 & 0.894427 & 0.927839 \\
2 & 4.442883 & 4.491214 & 1.414214 & 1.580210 \\
\hline
\end{tabular}


TABLE 4

Values for period $T$ and displacement amplitude A of equation (5.12) for initial conditions $(4.2 b, 4.3 b, 4.4 b)$ as given by formulae (4.5) and (4.6) with harmonic balance (harm. bal.) equation (5.13), for a range of values of initial velocity amplitude B, together with exact values obtained by numerical computation.

A

B harm. bal. exact harm. bal. exact

\begin{tabular}{lllll}
\hline .1 & 6.2753264 & 6.2753338 & 0.099875 & 0.099917 \\
.2 & 6.251690 & 6.251809 & 0.198997 & 0.199334 \\
.5 & 6.083668 & 6.088449 & 0.484123 & 0.489651 \\
1 & 5.441398 & 5.527200 & 0.866025 & 0.919107 \\
1.5 & 4.155936 & 4.690247 & 0.992157 & 1.240150 \\
\hline
\end{tabular}




\section{APPENDIX A}

The compatibility of equation (5.8) with equation (5.10) in the limit as the parameter $\gamma$ tends to zero is detailed here. For $\gamma$ small, in equation (5.10),

$$
\begin{aligned}
\cos \left(\sqrt{\gamma} \mathrm{x}+\mathrm{C}_{1}\right) & =\cos (\sqrt{\gamma} \mathrm{x}) \cos \left(\mathrm{C}_{1}\right)-\sin (\sqrt{\gamma} \mathrm{x}) \sin \left(\mathrm{C}_{1}\right) \\
= & \cos \left(\mathrm{C}_{1}\right)-\sqrt{\gamma} \mathrm{x} \sin \left(\mathrm{C}_{1}\right)+\mathrm{O}(\gamma) \\
= & -\sqrt{\gamma} \sin \left(\mathrm{C}_{1}\right)\left(\mathrm{x}-(1 / \sqrt{\gamma}) \cot \left(\mathrm{C}_{1}\right)\right)+\mathrm{O}(\gamma) \\
\therefore \ln \left|\cos \left(\sqrt{\gamma} \mathrm{x}+\mathrm{C}_{1}\right)\right| & \sim \ln \left|\mathrm{x}-(1 / \sqrt{\gamma}) \cot \left(\mathrm{C}_{1}\right)\right|+\ln \left|\sqrt{\gamma} \sin \left(\mathrm{C}_{1}\right)\right| .
\end{aligned}
$$

Thus, as $\gamma \rightarrow 0$,

$$
\mathrm{c}_{1} \sim-(1 / \sqrt{\gamma}) \cot \left(\mathrm{C}_{1}\right)
$$

and

$$
\mathrm{c}_{2} \sim \mathrm{C}_{2}+\ln \left|\sqrt{\gamma} \sin \left(\mathrm{C}_{1}\right)\right|
$$

in equation (5.8).

There is a $\gamma$-dependent relationship between the constants. The singularity of the relationship is not surprising since there is a transition from closed phase plane curves to open curves. This will not be pursued further here, since Section 5.2 is essentially concerned with the periods of solutions to the parameterless equation (5.12). 